\title{
NOVEDADES EN EL GÉNERO SIDA (MALVACEAE, TRIBU MALVEAE)
}

\author{
ANTONIO KRAPOVICKAS ${ }^{1}$
}

Summary: Krapovickas, A. 2007. Novelties in the genus Sida (Malvaceae, tribe Malveae). Bonplandia 16(3-4): 193-208. ISSN: 0524-0476.

The new species Sida Charpinii, S. Leitaofilhoi, S. pseudorubifolia, S. teresinensis and S. Tressensiae are described, the new name S. Honoriana is proposed and S. lonchitis is mentioned for the first time for Argentina and S. setosa for Paraguay.

Key words: Malvaceae, Sida, taxonomy.

Resumen: Krapovickas, A. 2007, Novedades en el género Sida (Malvaceae, tribu Malveae). Bonplandia 16(3-4): 193-208. ISSN: 0524-0476.

Se describen las especies nuevas Sida Charpinii, S. Leitaofilhoi, S. pseudorubifolia, S. teresinensis y S. Tressensiae, se propone el nuevo nombre S. Honoriana y se cita por primera vez a S. Ionchitis para Argentina y S. setosa para Paraguay.

Palabras clave: Malváceas, Sida, taxonomía.

\section{Sección Sida}

La Sección Sida se caracteriza por sus mericarpos 6-13, por las hojas con el margen dentado en los 2/3 superiores, liso en la base y por el cáliz con 10 nervios sobresalientes y más marcados en la base.

Las especies del cono sur de Sudamérica se pueden distinguir por medio de la siguiente clave.

A. Hojas farinosas. Cáliz sin pelos largos. Corola amarilla. Mericarpos 3 x 2,8 mm. 2n=14.

S. rhombifolia L.

A’. Hojas pubescentes. Cáliz con pelos largos.

B. Mericarpos múticos.

C. Flores en glomérulos axilares, pedúnculos 3-5 mm long. Perú. Ecuador.

S. Weberbaueri Ulbr.

C’. Flores solitarias, pedúnculos 2-4 cm long. 2n=14. Brasil (Minas Gerais).

S. Leitaofilhoi Krapov.

B'. Mericarpos aristados.

${ }^{1}$ Instituto de Botánica del Nordeste (UNNE-CONICET), Casilla de Correo 209, 3400 Corrientes, Argentina. E-mail: ibone@agr.unne.edu.ar 
D. Aristas hasta el doble que el cuerpo del mericarpo. Norte de Sudamérica, hasta Paraguay, Panamá.

S. setosa Mart. ex Colla

D’. Aristas más cortas que el cuerpo.

E. Flores agrupadas en el ápice de las ramas, brácteas bifidas presentes.

F. Mericarpos con aristas iguales o algo más cortas que el cuerpo. Epifilo por lo común con pelos simples. Sur de Brasil, NE de Argentina.

S. Honoriana Krapov.

F’. Mericarpos con aristas muy breves. Haz con pelos estrellados pequeños. $2 n=14$. Sur de Brasil.

S. lonchitis A.St.-Hil. \& Naudin

E’. Flores en glomérulos axilares, sin brácteas bífidas.

G. Cáliz acrescente, en fruto 9 mm x 6 mm. 2n=14.

S. santaremensis Monteiro

G’. Cáliz no acrescente.

H. Follaje amarillento, dorado. $2 \mathrm{n}=28$.

S. Glaziovii K.Schum.

H'. Follaje verde.

I. Indumento del cáliz sólo con pelos simples en el margen de los lóbulos. 2n=28.

S. tuberculata R. E. Fr.

I’. Indumento del cáliz con pelos simples en la base. $2 \mathrm{n}=14$.

S. Poeppigiana (K. Schum.) Fryxell

1. Sida Honoriana Krapov., nom. \& stat. nov.

Fig. 1

Sida rhombifolia L. var. surinamensis (Miq.) K. Schum. f. Sampaioi Monteiro, O genero Sida, Revisão das especies brasileiras: 46, tab. XI. 1936. Typus: Brasil, Rio de Janeiro, Therezopolis, 21III-1917, Sampaio 1965 (holotypus R 27854!) [non S. Sampaiana Monteiro] (CINB, Recomendación 23A.2).

Sida rhombifolia L. var. surinamensis (Miq.) K. Schum. f. corchorifolia Monteiro, O genero Sida, Revisão das especies brasileiras: 46, tab. II, fig. 8. 1936. Typus: Brasil, Rio de Janeiro, Nova Friburgo, 24-VIII-1935, Monteiro 1200 (holotypus RBR!) [non S. corchorifolia Tenore].

Sida kohautiana Presl var. Sampaioi (Monteiro) Monteiro, Annais XXIII Congr. Soc. Bot. Brasil: 134. 1972.

Sida kohautiana Presl var. corchorifolia
(Monteiro) Monteiro, Annais XXIII Congr. Soc. Bot. Brasil: 134. 1972.

Floribus solitariis vel glomeratis, ad apicen caulibus congesti. Bracteis bifidis. Carpidiis aristatis, aristis quam carpidiis longioribus.

Subarbusto perenne, erecto, 1-2 m alt. Tallo con pelos estrellados diminutos y pelos simples 0,5 mm long., esparcidos. Estípulas lineares, 4-5 mm long. Pecíolo 10-15 mm long., con pelos estrellados muy pequeños y pelos simples $0,5 \mathrm{~mm}$ long. más o menos densos. Lámina ovada, hasta 13,5 cm x $5 \mathrm{~cm}$, margen dentado en los 2/3 superiores, haz con pelos simples pequeños o con pelos estrellados pequeños, envés densamente cubierto de pelos estrellados y con algunos pelos simples $0,5 \mathrm{~mm}$ long. Flores solitarias o en glomérulos axilares. Pedúnculo 7-15 mm long., articulado en el medio. Cáliz $6 \mathrm{~mm}$ long., lóbulos triangulares $3 \mathrm{~mm}$ x $3 \mathrm{~mm}$, con 
A. Krapovickas, Novedades en el género Sida (Malvaceae)

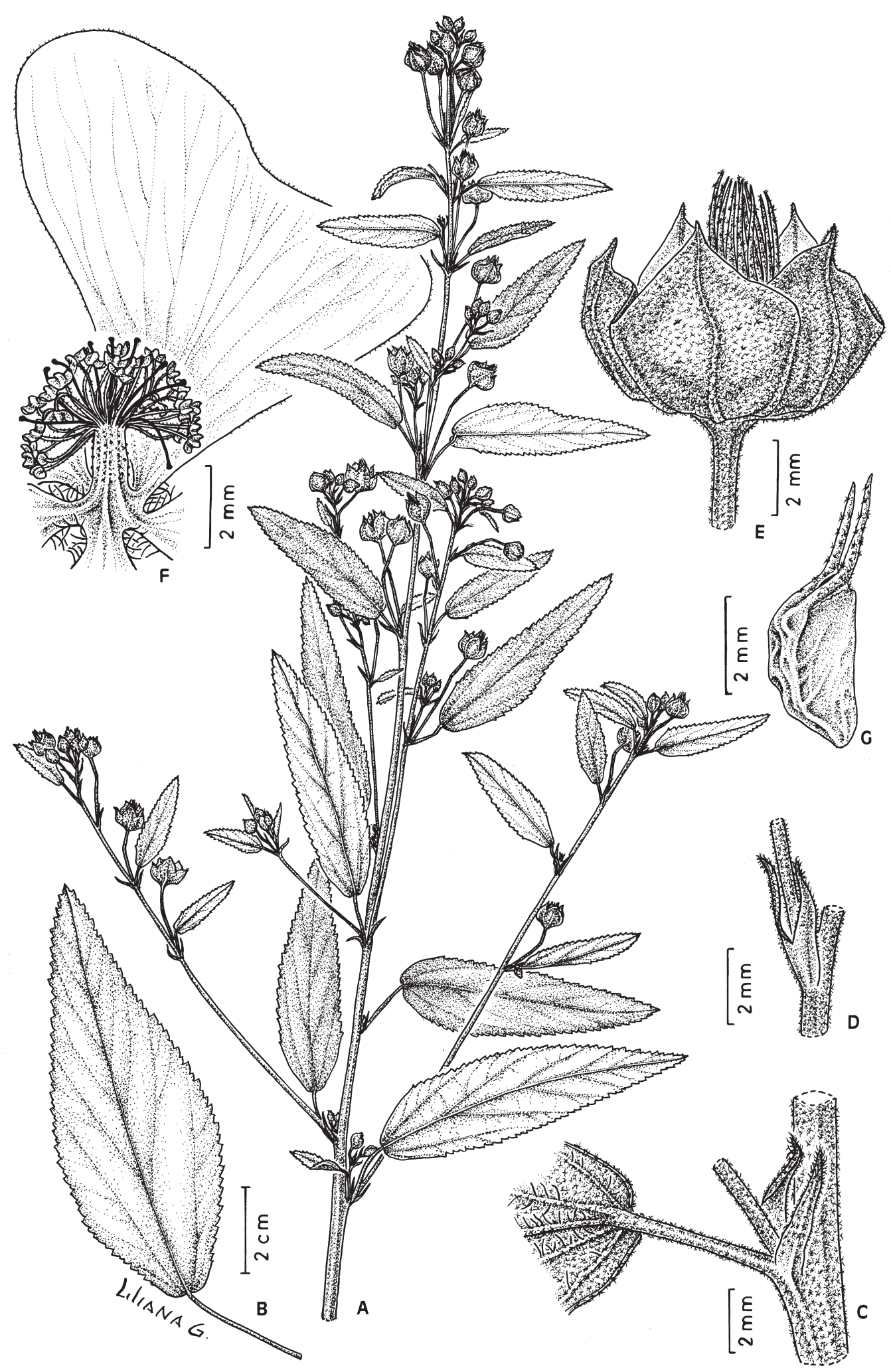

Fig. 1. Sida Honoriana Krapov. A: rama. B: hoja. C: estípulas. D: bráctea bífida de la inflorescencia. E: cáliz. F: pétalo y tubo estaminal. G: mericarpo (A-G, Krapovickas \& al. 35500). 
10 nervios gruesos en la base, solamente con pelos estrellados muy pequeños. Corola amarilla o anaranjada. Carpelos 9-10. Mericarpos $3 \mathrm{~mm}$ long. $\mathrm{x} 2 \mathrm{~mm}$ lat., con 2 aristas apicales 2,5-3 mm long., ápice con pelos estrellados pequeños y las aristas con pocos pelos estrellados, algo antrorsos.

Material adicional estudiado: ARGENTINA. Misiones. Dep. Guaraní, Predio Guaraní, UNaM, 12-II-2002, Keller \& Robledo 1604_(CTES); Parque Provincial Esmeralda, 16-VI-2003, Keller \& al. 2153 (CTES, SI, TEX); Reserva de Biosfera Yabotí, Mesa Redonda, 15-XII-2004, Keller 2856 (CTES). BRASIL. Distrito Federal. Brasilia, Horto do Guará, 10-III-1961, Heringer 8130/324 (CTES). Minas Gerais. Mun. Ipuiuna, Ipuiuna, 27-I-1980, Krapovickas \& Cristóbal 35500 (CTES, MBM, SP, TEX); Mun. Paraopeba, Faz. Da Onça, 26-V-1957, Heringer 5599 (CTES). Paraná. Mun. Agua Amarela, Rod. do Xisto, 17-IV-1973, Hatschbach 31814 (CTES, MBM); Mun. Campo Largo, Serra Javacaem, 25-IV-1971, Imaguire 486 (CTES, MBM); Mun. Curitiba, Capão da Imbuia, 28-I-1973, Imaguire 749 (CTES, MBM); Mun. Rio Branco do Sul, Serra do Votuvoru, 30-IV1974, Hatschbach 34404 (CTES, MBM); Curiola, 12-III-1967, Hatschbach 16151 (CTES, MBM); Mun. Campo Magro, Conceição dos Tulios, 25-II2003, Ribas \& al. 5126 (CTES, MBM). São Paulo. São Paulo, Barr. da Cantareira, 16-IV-1944, W. Hoehne 3450 (CTES, SPF); Cantareira, 16-IV1944, W. Hoehne 13450 (CTES, SPF); Terrenos F.M.S.P. 3-VI-1932, W. Hoehne 17338 (CTES, SPF); Jaraguá, 3-III-1932, W. Hoehne 13449 (CTES, SPF); Mun. Cabreuva, Cabreuva, mata da Casa Branca, 17-III-1994, Barreto \& al. 2185 (CTES, SP); Mun. Moji das Cruzes, Fazenda do Parataí, 21II-1941, Pickel 5259 (IPA); Mun. Cunha, Parque Estadual da Serra do Mar, 29-III-1994, Baitello 541 (CTES); ídem, 19-III-1996, Rapini \& al. 85 (CTES, SP); Mun. Guapiara, Fazanda Intervales, 26-IV1995, Kirizawa \& al. 3062 (CTES, SP); Monte Alegre do Sul, Estação Experimental do IAC, 15-III1995, Bernacci \& al. 1243 (CTES, IAC); São Roque, Estação Experimental do IAC, 24-IV-1995, Bernacci \& al. 1434 (CTES, IAC); Sete Barras, Parque Estadual de Carlos Botelho, 21-IV-2002, Savassi \& al. 268 (CTES, ESA).

Obs. 1: Dedico esta especie a Honorio da
Costa Monteiro Filho autor de la forma Sampaioi. Ya existen Sida Sampaiana Monteiro, 1967 y Sida corchorifolia Tenore, 1857.

Obs. 2: Sida honoriana pertenece a la sección Sida por sus hojas rómbicas, en disposición espiralada, con el margen crenado-aserrado hacia el ápice y liso hacia la base. Es afín a S. setosa Mart. ex Colla por las aristas de los mericarpos muy desarrolladas. Se diferencia de ésta porque en $S$. setosa (Fig. 2 D-G) las aristas son mucho más largas, 6-7 mm long., hasta 2 veces el largo del mericarpo, las hojas solo tienen pelitos estrellados punctiformes muy espaciados en ambas caras, pedúnculos 6-22 $\mathrm{mm}$ long.

\section{Sida lonchitis A.St.-Hil. \& Naudin}

Fig. 2 A-C

Saint Hilaire, A. \& C. V. Naudin, Ann. Sci. Nat. Bot., ser. 2, 18: 50, 1842. Typus: "Prope Rio de Janeiro legit Gaudichaud.- Herb. Mus. Par.". Lectotypus, aquí designado: Bresil, Rio de Janeiro, Gaudichaud 938 [P!, isotypus Gaudichaud 938, 1831-33 (Herbier E. Drake) (P!, foto F 35533!].

Sida incerta A.St.-Hil. \& Naudin, Ann. Sci. Nat. Bot. ser 2, 18: 51. Typus: "Prov. Minas Geraes" [Holotypus: Bresil, prov. Minas Geraes (Herb. Impérial Bresil no 95), Herb. Mus. Paris, $C$. Gaudichaud 1833 P!]. Nuevo sinónimo aquí propuesto.

Subarbusto 0,40 a $1,50 \mathrm{~m}$ alt. Tallo con pelos estrellados muy pequeños que dejan ver la epidermis. Estípulas angosto-triangulares, 2-5 mm long. Pecíolo 5-6 mm long., con indumento similar al del tallo. Lámina ovada, hasta $10 \mathrm{~cm}$ long. $x 4 \mathrm{~cm}$ lat., margen dentado en los 2/3 superiores, haz con pelos estrellados diminutos esparcidos, envés densamente cubierto de pelos estrellados algo mayores, blanquecinos. Flores agrupadas en el ápice de las ramas, en la axila de brácteas bífidas. Pedúnculo articulado, 10-12 mm long. Cáliz 6 mm long., lóbulos triangulares $3 \mathrm{~mm}$ long. x $3 \mathrm{~mm}$ lat., con pelos estrellados diminutos. Corola amarilla sin mancha basal, a veces blanca. 

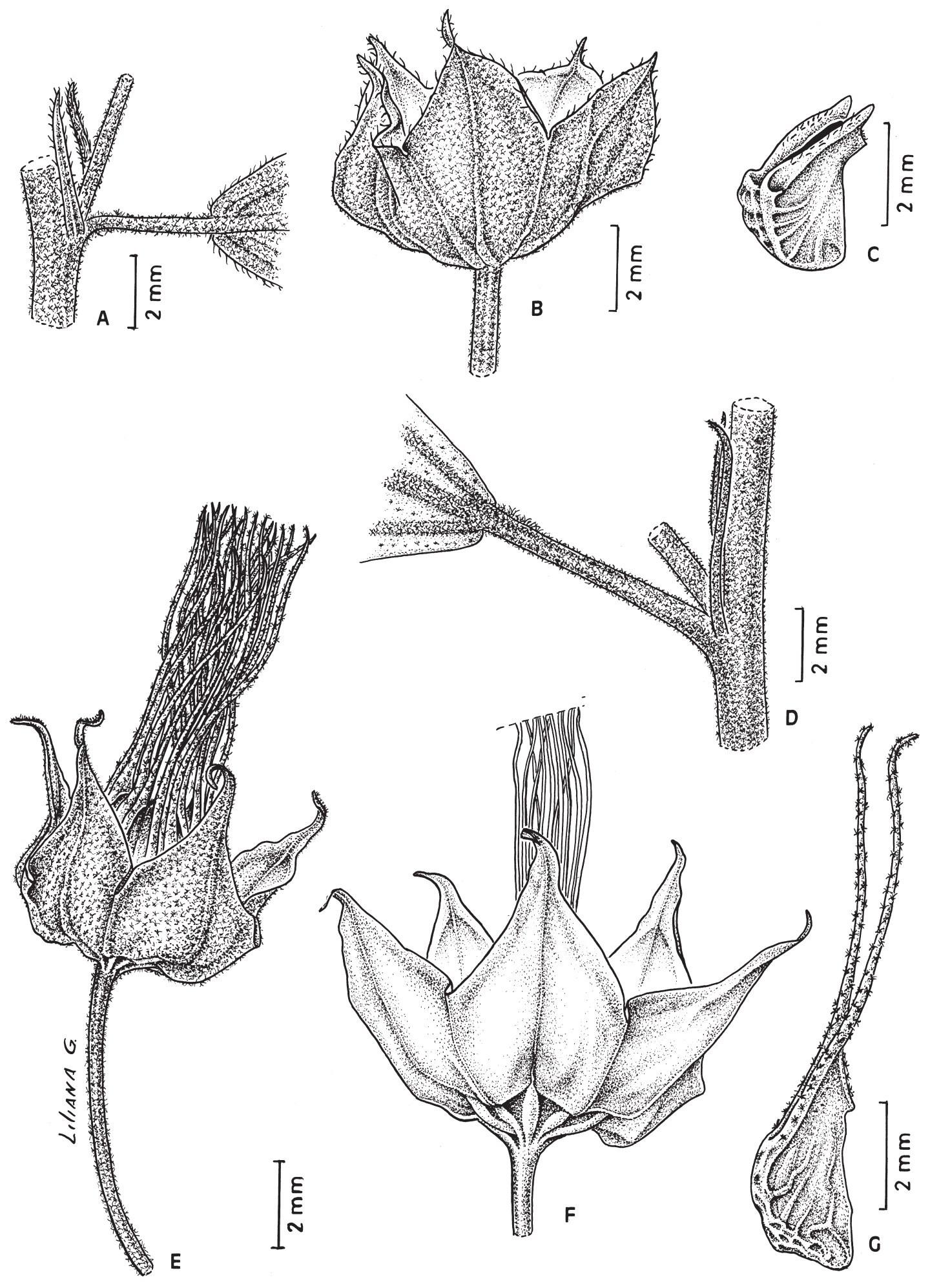

Fig. 2. A-C, Sida lonchitis. A: estípulas. B: cáliz. C: mericarpo (A-C, Krapovickas \& al. 23243). D-G, S. setosa. D: estípulas. E: cáliz y aristas del fruto. F: base del cáliz sin pubescencia. G: mericarpo (D-G, Ferreyra 9295). 
Carpelos 10-11. Mericarpos 3 mm long. x 2 $\mathrm{mm}$ lat., submúticos o con dos aristas $0,3 \mathrm{~mm}$ long., con pelos muy pequeños en el ápice. Cromosomas 2n=14 (Fernández, 1981).

Material seleccionado estudiado: BRASIL. Distrito Federal. Brasilia, 25-XI-1965, Irwin \& al. 10710 (CTES, NY). Minas Gerais. Bandeira do Sul, 20-I-1980, Krapovickas \& Cristóbal 35400 (CTES, F, ICN); Poços de Caldas, 19-I-1980, Krapovickas \& Cristóbal 35392 (CTES, MBM, MEXU). Paraná. Mun. Guarapuava, Canta Galo, 7-II-1969, Hatschbach 21042 (CTES, MBM); Mun. Morretes, Col. Floresta, 23-I-1969, Hatschbach 20875 (CTES, MBM). Rio de Janeiro. Estrada Rio-Petropolis, km 30, 12-II-1973, Krapovickas \& al. 23243 (CTES, MBM, SP, TEX); Estrada Friburgo-Teresópolis, km 80, 11-I-1973, Occhioni 5421 (CTES, RFA); Parque Nacional Itatiaia, km 10, 7-III-1947, Occhioni 849 (CTES, RFA). Santa Catarina. Porto Belo,8-II1983, Krapovickas \& cristóbal 38444 (CTES, HAS, LIL, MBM ); Três Barras, Garuva, 21-I-1958, Reitz \& Klein 6248 (CTES, HBR); Curitibanos, 22-II1962, Reitz \& Klein 12263 (CTES, HBR); Cunhas, Itajaí, 4-I-1955, Klein 955 (CTES, HBR); Penha, 17I-1985, Krapovickas \& Cristóbal 39609 (CTES, C, MBM, MO). São Paulo. São Paulo, 20-I-1968, Gottsberger 11-20168 (CTES); São Paulo, 5-II1968, Gottsberger 11-5268 (CTES); Mongaguá, 29XII-1953, Bartolomeu 5198 (CTES, SPF).

Obs.: Esta especie figura en los herbarios generalmente como Sida rhombifolia L., de la que se diferencia por el tomento, por sus flores agrupadas en el ápice de las ramas y por la presencia de brácteas bífidas en la base de las flores.

\section{Sida setosa Mart. ex Colla}

Fig. 2 D-G

Iconografía. Fryxell, 1992: 111, fig. 12.

Material estudiado: PERÚ. Amazonas. Huambisa, Valle del Rio Santiago, 200 m, 20-XII1979, Tunqui 380 (CTES, MO). Huánuco. cerca á Puerto Nuevo, Tulumayo, en Tingo María, 600700 m, 20 VI-1953, Ferreyra 9295 (CTES, USM). PARAGUAY. Alto Paraná. Puerto Palma, 3-VII1980, Itaipú Binacional 811 (MO).

\section{Sida Leitaofilhoi Krapov., sp. nov.}

Fig. 3

Suffrutex 1-2 m altus. Foliis breviter petiolatis. Lamina rhombeo-lanceolata, serrata, basin versus integerrima, utrinque pilis stellatis. Floribus solitariis, longe pedunculatis. Calyce pyramidato, plicato, nervis 10 basi noduloso-incrassatis, utrinque pilis stellatis, ad basin et marginibus loborum pilis simplicibus longiusculis instructo. Carpidiis 7-8, submuticis.

Holotypus: Brasil, Minas Gerais, Mun. Santa Barbara, nativo em matas e campos da Serra do Caraçá, subarbusto até 1-2 m, flores amarelas, 13XII-1978, H. F. Leitão Filho, G. Shepherd \& W. Stubblebine \& al. 9649 (UEC, isotypi CTES, SP).

Subarbusto 1-2 m de altura, amarillento. Tallo velutino, completamente cubierto de pelos estrellados muy pequeños. Estípulas lineales, 6-11 mm long., adosadas al tallo. Lámina oval-lanceolada, hasta $7 \mathrm{~cm}$ long. x 1,5 cm lat., ápice agudo, margen aserrado y hacia la base liso, haz verde-amarillento, con indumento de pelos estrellados diminutos, envés amarillento, cubierto totalmente de pelos estrellados mayores. Flores solitarias, axilares, pedúnculo 2-4 cm long., articulado, con indumento igual al del tallo. Cáliz 7-8 mm long., velutino, en la base 10 nervios marcados, márgenes de los lóbulos plegados, lóbulos triangular-acorazonados, $5 \mathrm{~mm}$ long., $5 \mathrm{~mm}$ lat. en el centro y $3 \mathrm{~mm}$ lat. en la base, cubierto de pelos estrellados y con algunos pelos simples largos en la base del cáliz y en el extremo de los lóbulos. Corola amarilla. Pétalos 10-12 mm long., uña pilosa. Tubo estaminal glabro, $3 \mathrm{~mm}$ long. Estigmas en cabezuela, Carpelos 7-8. Mericarpos $4 \mathrm{~mm}$ long. x 2,5 mm lat., submúticos, con dos aristas apicales muy breves, dorso reticulado, ápice con pelos estrellados pequeños. Cromosomas 2n=14 (Lavia \& al., 2007).

Distribución geográfica: Vive en áreas montañosas, entre 800 y 1500 m de altura, en la Serra do Espinhaço, en el centro de Minas Gerais, Brasil. 
A. Krapovickas, Novedades en el género Sida (Malvaceae)

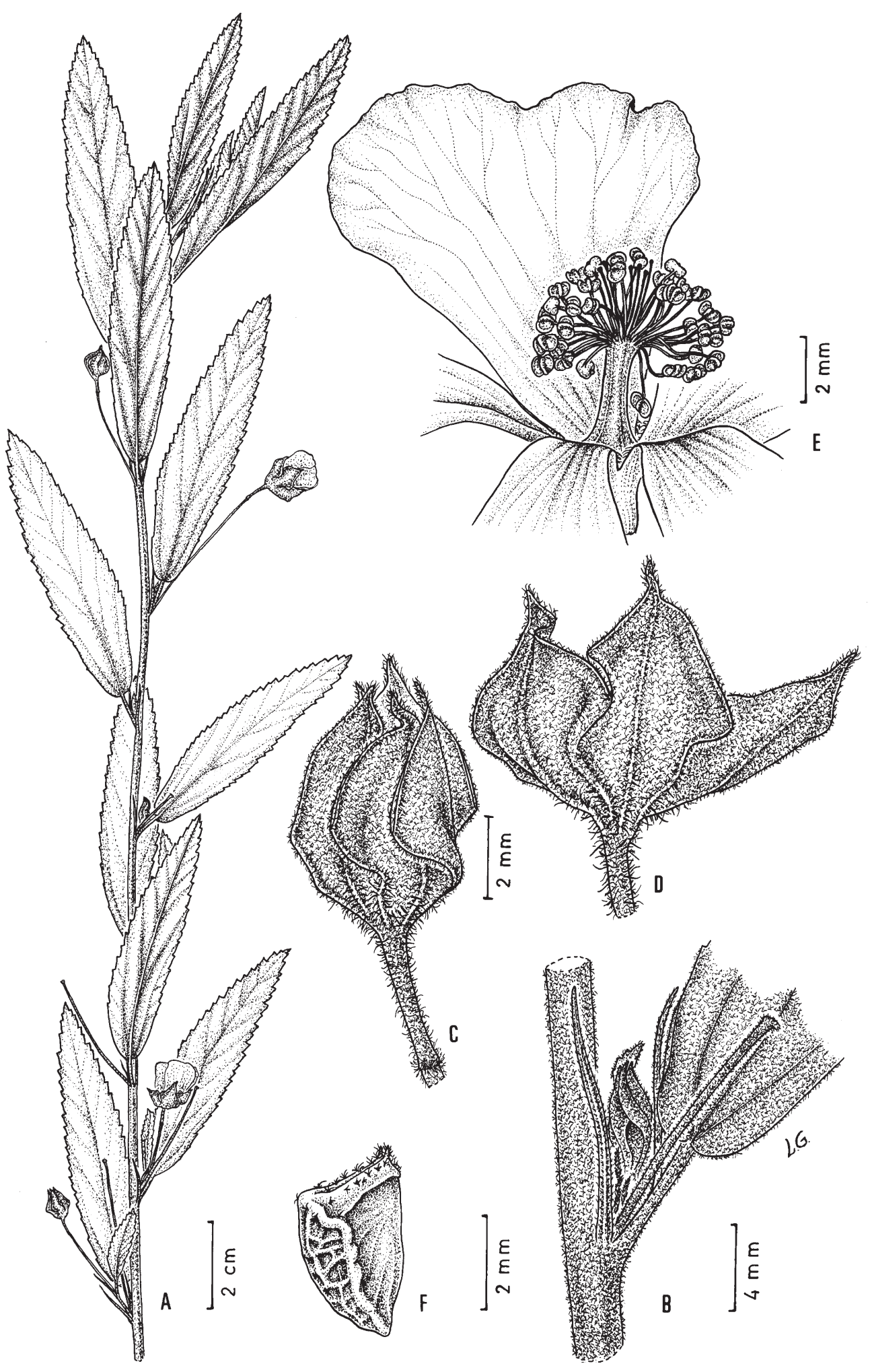

Fig. 3. Sida Leitaofilhoi Krapov. A: rama. B: base de la hoja. C-D: cáliz. E: pétalo y tubo estaminal. F: mericarpo (AF, Leitão Filho 9649). 
Paratypus: BRASIL. Minas Gerais. Barão de Cocais, Picha do cavalo, Mina Brucutu/CVRD, 1953'S, 4326' W, 26-III-2002, Oliveira 73 (BHCB, CTES); Rancharia, 15 km S de Ouro Preto, camino a Santa Rita de Ouro Preto, 1260 m, 13-V-1990, Arbo \& al. 4012 (CTES, NY, SPF); Mun. Ouro Preto, Morro do Cachorro, Serra Itacolomí, 1500 m, 4-II-1978, Krapovickas \& Cristóbal 33449 (CTES).

\section{Sección Cordifoliae (DC.) Fryxell}

\section{Sida Charpinii Krapov., sp. nov.}

Fig. 4

Herba 30-70 cm alta. Caulibus stellato subtomentosis pilisque simplicibus inspersis. Lamina ovata, serrata, supra stellato subtomentosa, subtus plus minus stellato tomentosa et insuper pilis simplicibus longiusculis inspersis. Floribus solitariis in axillis foliorum, prope apicem caulibus congesti. Calyx villoso. Tubo stamineo piloso. Carpidiis 7-9, reticulatis, submuticis. Semina glabra.

Holotypus: Paraguay, Boquerón, 8 km NW de Nueva Asunción ruta Transchaco, dunas consolidadas, erecta. 14-V-1994, A. Krapovickas, C. L. Cristóbal \& A. Schinini 45403 (CTES, isotypi FCQ, K, LPB NY, SI).

Hierba o sufrútice 30-70 cm de altura. Tallo con indumento de pelos estrellados y glandulares formando una capa densa y pelos simples 2-3 mm long., esparcidos. Estípulas filiformes 5-7 mm long., hirsutas. Pecíolo breve, 10-15 mm long. con indumento similar al del tallo. Lámina ovada, margen aserrado, haz con pelos estrellados que dejan ver la epidermis y con pelos glandulares en las hojas jóvenes; envés algo más claro con pelos estrellados densos, pelos glandulares y pelos simples largos dispersos. Flores solitarias. Pedúnculo articulado, 5-7 mm long. Cáliz 7-8 mm long., lóbulos triangulares $5 \mathrm{~mm}$ long. x $3 \mathrm{~mm}$ lat., cara externa cubierta de pelos estrellados y glandulares y además pelos simples 2-3 mm long. sobre los nervios; cara interna con pelos simples adpresos en el ápice de los lóbulos.
Pétalos $8 \mathrm{~mm}$ long. x 6,5 mm lat., glabros. Tubo estaminal $3 \mathrm{~mm}$ long., con pelos simples. Estilos 7-9. Carpelos 7-9, uniovulados. Mericarpos $4 \mathrm{~mm}$ long. x 2,5 mm lat., submúticos, dorso y caras laterales reticuladas y con pocos pelos en el ápice. Semilla péndula, glabra. Cromosomas 2n=14 (Lavia \& al., 2007).

Distribución geográfica: Vive en el oeste del Chaco paraguayo, en médanos consolidados y en los valles intermontanos del sur de Bolivia, entre 2000 y 3000 m de altura.

Obs.: Esta especie se parece mucho por su aspecto general a $S$. variegata (Griseb.) Krapov. Se diferencia por sus mericarpos submúticos en contraposición con los mericarpos con dos aristas muy largas en $S$. variegata.

Paratypi. BOLIVIA. Chuquisaca. Prov. Yamparaez, Sucre, ca. $15 \mathrm{~km}$ hacia Tarabuco, 3000 m, 7-III-1981, Beck 6208 (LPB). Cochabamba. Prov. Cercado, Zona Villa Pagador, laderas del cerro Abra, 2650 m, 2-III-2000, Barco 548 (CTES). Tarija. Prov. Arce, 63 km de Tarija hacia Bermejo, 2300 m, 24-I-1988, Ehrich 394 (LPB); Prov. Méndez, Tomatas Grande, 1950 m, 13-II1986, Bastián 763 (CTES, LPB). PARAGUAY. Alto Paraguay. 6,5 km SE de Tte. Gabino Mendoza, 366 m, 22-V-2003, Pérez de Molas \& Navarro 9227 (CTES, PY). Boquerón. Gral. Eugenio A. Garay, picada a S de la Delegación de Gobierno de Nueva Asunción, 350-400 m, 10-V1988, Charpin \& Ramella 21543 (CTES, G); 25 km SE de Nueva Asunción, 15-V-1994, Krapovickas \& al 45470 (CTES); 12 km NW de Nueva Asunción, 14-V-1994, Krapovickas \& al. 45534 (CTES); between Parque Nacional Teniente Agripino Enciso and Nueva Asunción, 2054’S 6150’W, 27-I-1995, Zardini \& al. 42391 (CTES, MO).

\section{Sida teresinensis Krapov., sp. nov.}

Fig. 5

Herba annua 40-60 cm alta. Caulibus stellato subtomentosis, pilibus stellatis majoribus inspersis. Lamina ovata, crenata, supra pilis stellatis parvis inspersis vel subglabra, infra 
A. Krapovickas, Novedades en el género Sida (Malvaceae)

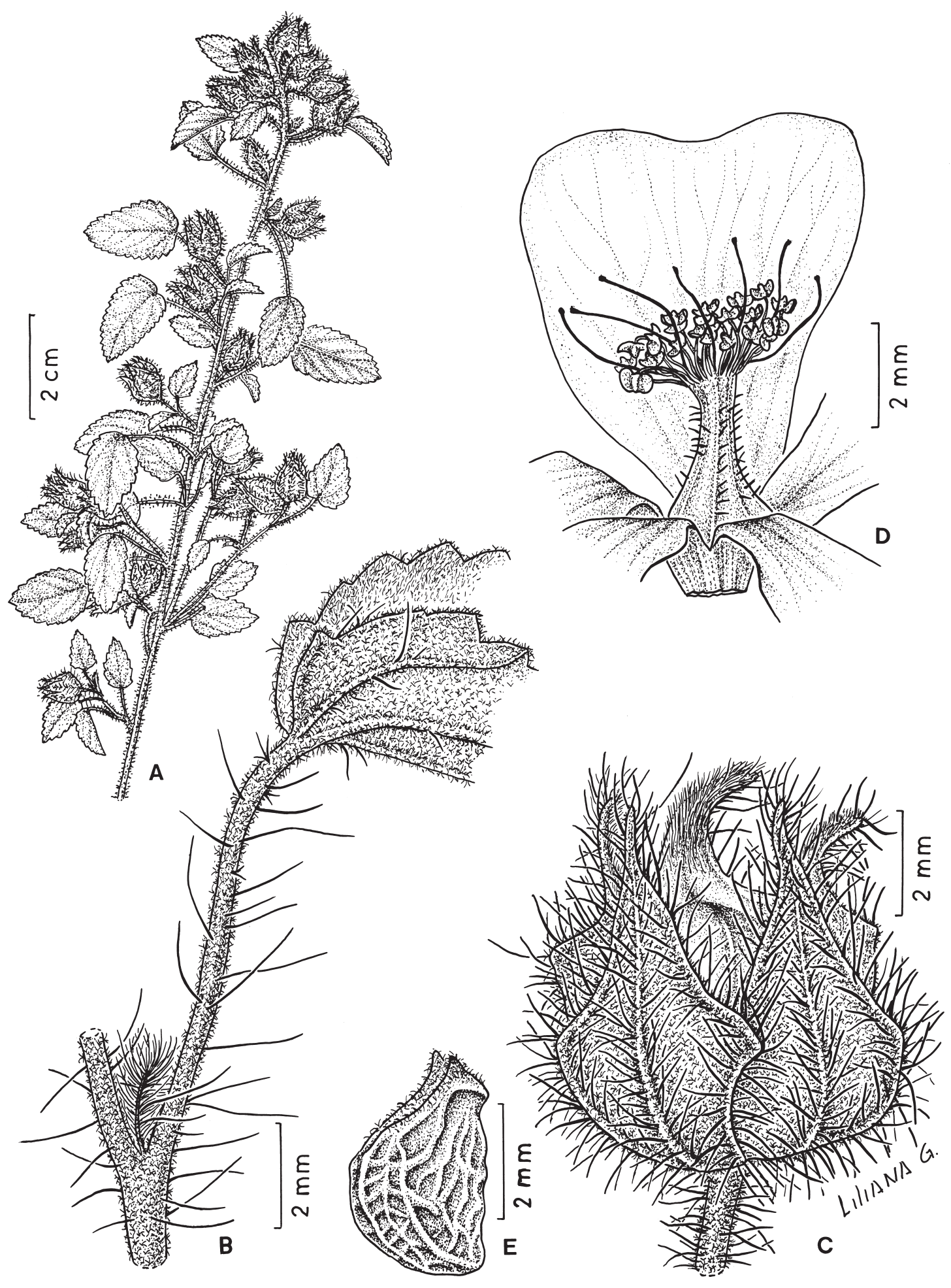

Fig. 4. Sida Charpinii Krapov. A: rama. B: pecíolo y estípulas. C: cáliz. D: pétalo y tubo estaminal. E: mericarpo (AE, Krapovickas 45470). 


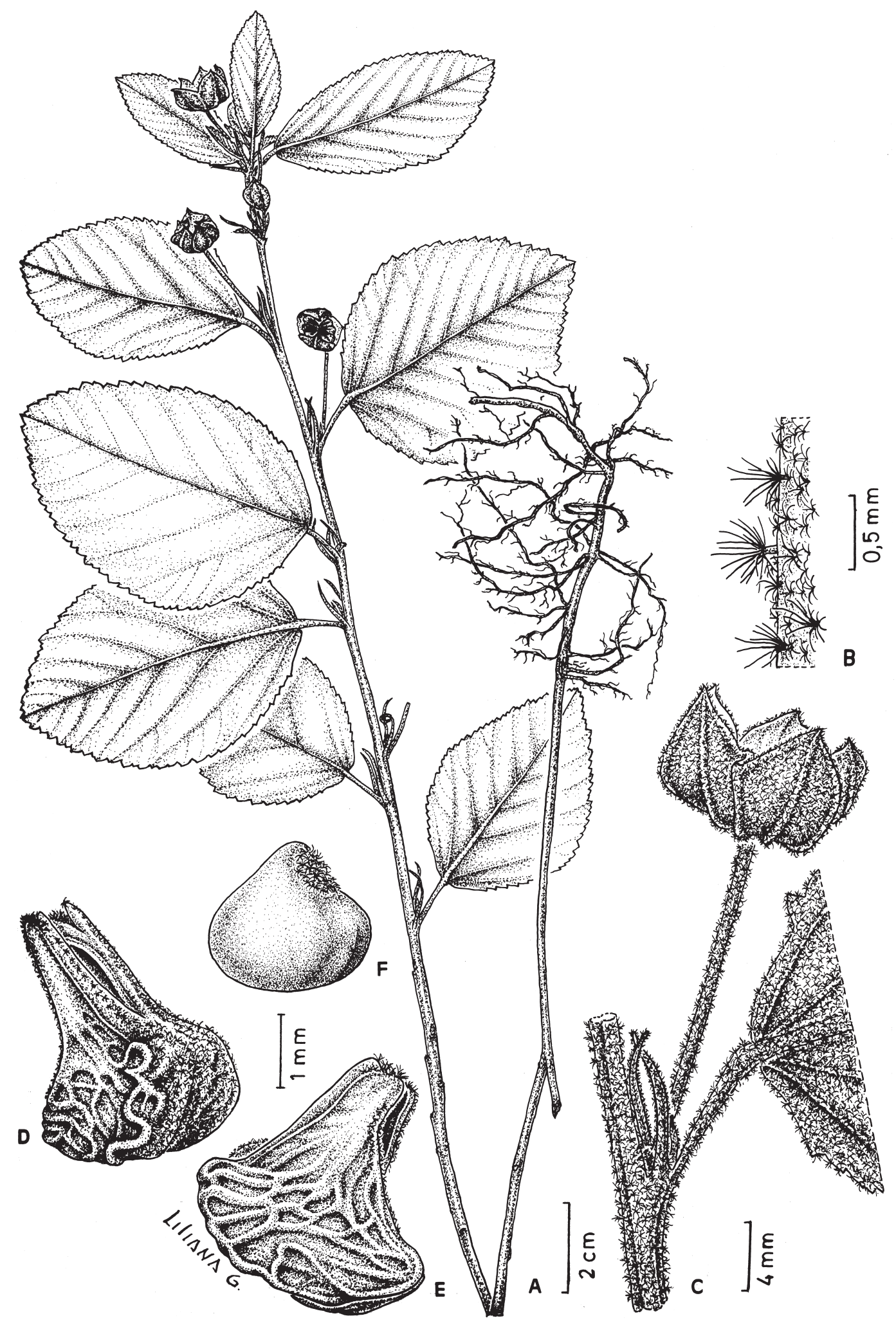

Fig. 5. Sida teresinensis. A: planta. B: indumento del tallo. C: estípulas y flor. D: mericarpo, vista 3/4. E: mericarpo, cara lateral. F: semilla (A-F, Krapovickas \& Cristóbal 37221, typus) 
stellato subtomentosa. Floribus solitariis in axilis foliorum. Calyx stellato tomentoso, nervis 10 incrassatis. Corola lutea. Carpidiis 13, reticulatis, mutici.

Holotypus: Brasil, Piahuí, Teresina, Parque Zoobotánico, flor amarilla, 31-I-1981, A. Krapovickas \& C. L. Cristóbal 37221 (TEPB, isotypi CTES, G, MBM, MO, SI, SP, TEX).

Planta anual, erecta, 40-60 cm altura. Tallo totalmente cubierto de pelos estrellados pequeños y con algunos pelos estrellados mayores, pedicelados, esparcidos Hojas alternas. Estípulas aleznadas, 8-10 mm long. x 0,5 mm lat., estrellado tomentosas. Pecíolo 7-10 mm long, estrellado tomentoso. Lámina anchamente oval, pentanervada, hasta 50 mm long. x $45 \mathrm{~mm}$ lat., margen suavemente crenado casi hasta la base; haz con pelos estrellados diminutos, esparcidos a subglabro; envés con indumento similar al del tallo. Flores solitarias, axilares, a lo largo de las ramas con pedúnculo $20 \mathrm{~mm}$ long. o en ramitas muy breves con pedúnculo 5 mm long. Pedúnculo no articulado, estrellado tomentoso. Cáliz 5-alado, estrellado tomentoso, $10 \mathrm{~mm}$ long., con 10 nervios prominentes basales; lóbulos $5 \mathrm{~mm}$ long. $\mathrm{x}$ 6-7 mm lat. Corola amarilla. Mericarpos 13, submúticos, $3 \mathrm{~mm}$ long x $3 \mathrm{~mm}$ lat., $3 / 4$ basal reticulado, indehiscente, porción superior lisa, dehiscente, con pelos estrellados diminutos, apenas visibles y con pelos blancuzcos en el ápice. Semilla glabra con pocos pelos estrellados en el hilo.

Paratypus: BRASIL. Pará. Belem, Murutucú, V-VI-1934, Insp. de Plantas Texteis $n^{\circ} 11$ (LP 74149).

Distribución geográfica: Vive en la reserva de bosque del Parque Zoobotanico de Teresina, en forma aislada, no constituyendo poblaciones.

Obs.: Pertenece a la sección Cordifoliae (DC) Fryxell. Es parecida a Sida cordifolia L. por la forma de las hojas, de la que se diferencia por el tomento, por sus flores solitarias y por sus mericarpos submúticos.

\section{Sida pseudorubifolia Krapov. \& Bueno, sp. nov.}

Fig. 6

Iconografía: Schumann, 1891, tab.61.

Sida rubifolia auct. non A.St.-Hil. (Schumann, 1891: 328-329).

Herba perennis. Caulibus pilis brevibus stellatis subtomentosis et simplicibus longiusculis plus minus hispidis. Stipulae 12-15 mm longae. Lamina ovato-oblonga, basi cordata vel truncata, crenato serrata. Floribus axillaribus solitaris longe pedunculatis. Calyce pyramidato plicatopentagono. Carpidiis 10, submutici.

Holotypus: Brasil, Rio grande do Sul, Tupanciretan, in campo, 29-I-1942, Rambo 9472 (PACA, isotypus LIL).

Hierba perenne. Tallo cubierto por una capa densa de pelos estrellados muy pequeños y con pelos simples, 1-2 mm long., abundantes. Estípulas lanceoladas 12-15 mm long. x 0,3-0,5 mm lat. Pecíolo 5-20 $\mathrm{mm}$ long., con indumento similar al del tallo. Lámina ovado-oblonga, 3-5 cm long. x 1,5-3 cm lat., base horizontal a subcordada, margen crenado-aserrado hasta la base, haz densamente cubierto de pelos simples 0,5-1 $\mathrm{mm}$ long., envés verde amarillento, cubierto de pelos estrellados con radios 0,5-1 mm long. Flores solitarias, axilares. Pedúnculo 1-2 cm long., con tomento similar al del tallo. Cáliz tomentoso, 12-15 mm long., lóbulos triangulares $6 \mathrm{~mm}$ long. x $6 \mathrm{~mm}$ lat., plegados. Pétalos $20 \mathrm{~mm}$ long., uña pilosa, cara externa con pelos simples diminutos, hialinos. Tubo estaminal $4 \mathrm{~mm}$ long., glabro. Carpelos 10. Mericarpos $4 \mathrm{~mm}$ long., 2,5 mm lat., submúticos, caras laterales reticuladas en la base, ápice y dorso con pelos estrellados diminutos.

Obs. 1: La ilustración de Flora brasiliensis se basa en el ejemplar Sellow 3110, que fue coleccionado en Rio Grande do Sul "da Encruzilhada ate Caçapava e o Rio Santa Barbara, XI-XII de 1825" (Urban, 1893: 186). 


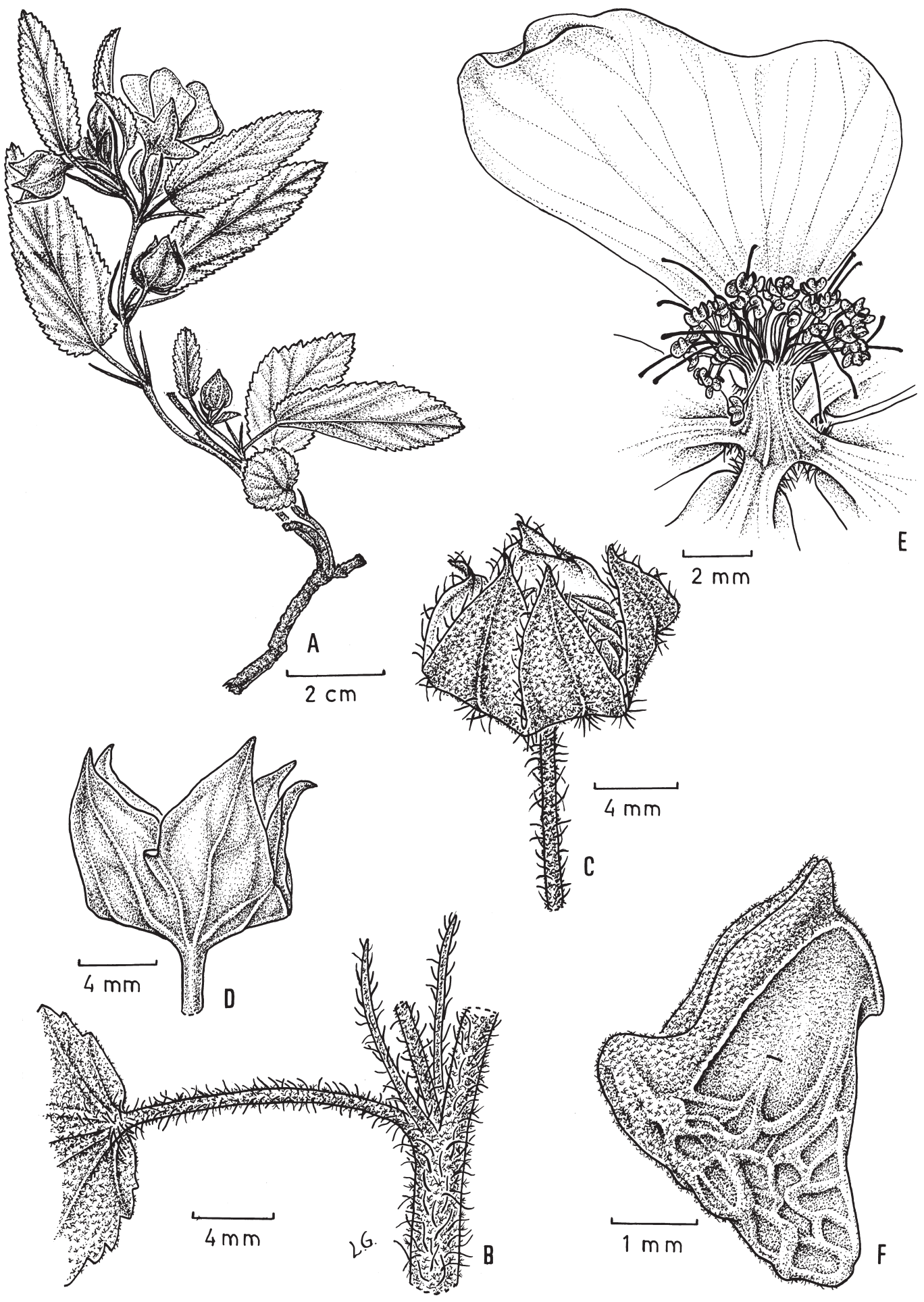

Fig. 6. Sida pseudorubifolia Krapov. A: rama. B: estípulas. C: cáliz. D: cáliz mostrando los nervios basales. E: pétalo y tubo estaminal. F: mericarpo (A-F, Arbo 2467). 
Obs. 2. Por sus hojas subcordadas y por tener 10 carpelos pertenece a la sección Cordifoliae.

Paratypi. BRASIL. Rio Grande do Sul. Jarí p. Tupanciretan, 4-X-1945, Rambo 9124 (PACA); Caçapava do Sul, 13-XII-1982, Falkennberg s. $n$. (CTES); São Francisco de Assis, 6 km de Manuel Viana, 5-XI-1981, J. Mattos 31280 (CTES, HAS); $20 \mathrm{~km}$ S de BR-290, camino a Santana da Boa Vista, 18-XII-1981, Arbo \& Schinini 2467 (CTES).

\section{Sida Tressensiae Krapov., sp. nov.}

Fig. 7

Herba perennis $50 \mathrm{~cm}$ alta. Caulibus stellato pubescens et pilis simplicibus $1 \mathrm{~mm}$ long. inspersis. Stipulae 4-5 mm long., lineari. Lamina ovata, basi subcordata, margine dentato, stellato pubescens. Floribus solitariis, axilaribus. Calyx pubescens, nervi 10, incrassati. Carpidii 8, pauce reticulati, mutici.

Holotypus: Argentina, Corrientes, dep. San Miguel, ea. San Juan Poriajhu, ruta 17, 18 km de ruta 12, potrero Tres Marías, en palmar de Butia paraguayensis, flores color crema, 8-XII-1992, S.G. Tressens, M. Beccaceci y R. Vanni 4552 (CTES, isotypi SI, TEX).

Hierba perenne hasta $50 \mathrm{~cm}$ de altura, pubescente, toda la planta cubierta con pelos estrellados muy pequeños, densos. Tallo estrellado-pubescente y con pelos simples 1 $\mathrm{mm}$ long. esparcidos, caducos, presentes en partes jóvenes. Estípulas 4-5 mm long. x 0,5-1 mm lat., uninervadas y con el margen ciliado; pecíolo 3-5 mm long.; lámina elíptica a algo ovada, de $30 \mathrm{~mm}$ x $20 \mathrm{~mm}$ a $40 \mathrm{~mm}$ x $15 \mathrm{~mm}$, base subcordada, ápice subagudo, margen dentado hasta la base, ciliado; epifilo por lo común pubescente, pero en hojas basales suele presentar pelos simples ca. $1 \mathrm{~mm}$ long., algo adpresos, hipofilo con pelos estrellados densos, algo mayores que los del epifilo. Flores solitarias, axilares, pedúnculo 25-40 mm long., articulado ca. $10 \mathrm{~mm}$ por debajo de la flor. Cáliz pubescente $10 \mathrm{~mm}$ long., con 10 nervios sobresalientes en la base, lóbulos triangulares $7 \mathrm{~mm}$ long. x $7 \mathrm{~mm}$ lat. margen ciliado, y márgenes de dos lóbulos contiguos replegados. Corola color crema, pétalos asimétricos, $15 \mathrm{~mm}$ long. x $15 \mathrm{~mm}$ lat., uña pilosa. Tubo estaminal $5 \mathrm{~mm}$ long., glabro, anteras apicales. Mericarpos 8, trígonos, múticos, glabros, $3 \mathrm{~mm}$ long. $\mathrm{x} 2 \mathrm{~mm}$ lat., 1 $\mathrm{mm}$ apical liso y $2 \mathrm{~mm}$ basales reticulados, tanto en la cara dorsal como en las dos laterales. Semilla péndula, glabra.

Distribución geográfica: Vive en el nordeste de la provincia de Corrientes en suelos arenosos con palmares de "yatay poñí" [Butia paraguayensis (Barb. Rodr.) Bailey].

Paratypi: ARGENTINA. Corrientes. Dep. Ituzaingó, isla Apipé Grande, ca. $5 \mathrm{~km}$ de Pto. Arazá, 26-XI-1988, Tressens \& al. 3500 (CTES); $8 \mathrm{~km} \mathrm{~S}$ de ruta 12 en camino vecinal a ea. San José (10 km S de Villa Olivari), 18-II-2002, Tressens, Vanni \& López 6711 (CTES).

Obs.1: Dedico esta especie a la Lic. Sara Graciela Tressens quién participó en la colección de todos los ejemplares mencionados.

Obs. 2: Por sus hojas subcordadas y por tener 8 carpelos pertenece a la sección Cordifoliae.

Obs. 3: Por sus flores solitarias con pedúnculos más largos que los pecíolos, por sus pétalos de más de $15 \mathrm{~mm}$ long, por los lóbulos del cáliz replegados y por los mericarpos múticos o submúticos se parece a S. pseudocymbalaria (Hassl.) Hassl., S. ascendens A.St.-Hil., S. rubifolia A.St.-Hil. y S. pseudorubifolia Krapov.

Estas especie se pueden separar por medio de la siguiente clave. 


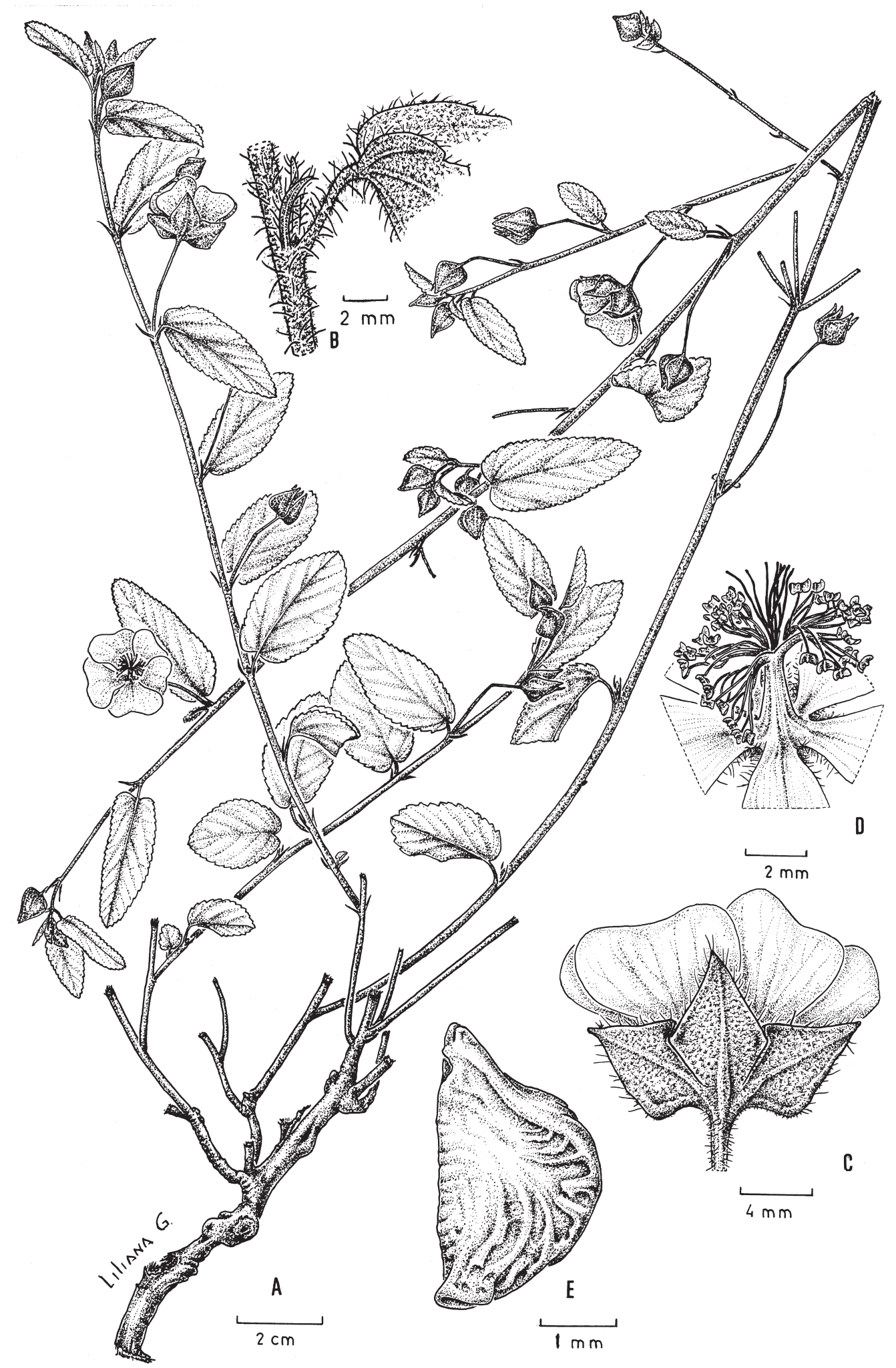

Fig. 7. Sida Tressensiae Krapov. A: planta. B: pecíolo y estípulas. C: cáliz y corola. D: tubo estaminal. E: mericarpo (A-E, Tressens 4252, holotypus). 
A. Mericarpos lisos. Corola blanca. São Paulo, Paraná (Brasil).

S. ascendens A.St.-Hil.

A'. Mericarpos reticulados.

B. Base del cáliz liso.

C. Planta procumbente. Corola blanco crema. Paraguay.

C’. Planta erecta. Corola amarilla. Minas Gerais (Brasil).

S. pseudocymbalaria (Hassl.) Hassl.

B’ Base del cáliz con 10 nervios prominentes.

S. rubifolia A.St.-Hil.

D. Mericarpos glabros. Estípulas 3-6 mm long. x 0,5-1 mm lat. Corola crema. Corrientes (Argentina).

S. Tressensiae Krapov.

D’. Mericarpos pubescentes. Estípulas filiformes 12-15 mm long. Rio Grande do Sul (Brasil).

S. pseudorubifolia Krapov.

\section{Sida ascendens A.St.-Hil.}

Saint Hilaire, A., Fl Bras. mer. 1: 182-183, 1827. Typus: "prope rivum Tareré [Itareré], in parte australi provinciae S. Pauli" (holotypus P!).

Sida pararaguariensis Hochr., in Chodat \& Hassler, Pl. Hassl. 2: 557, 1905. Typus: in arenosis Ipe hu, Sierra de Maracayú, Oct., Hassler 5121 (holotypus G!, isotypus K!).

Material adicional estudiado: BRASIL. Paraná. Mun. Guarapava, Cadeado, 14-XI-1973, Hatschbach 33530 (CTES, MBM); Mun. Laranjeiras do Sul, Laranjeiras do Sul, 890 m, 16XII-1966, Hatschbach 15536 (CTES, MBM). Santa Catarina, Abelardo Luz, 900 m, 29-XII1963, Reitz \& Klein 16567 (CTES).

\section{Sida rubifolia A.St.-Hil.}

Saint Hilaire, A., Fl Bras. mer. 1: 183-184, tab. 34, 1827. Typus: "prope praedium Itajuru haud longe a vico S. Miguel de Mato Dentro, in provincia Minas Geraes" (holotypus P!).

Obs.: El tipo fue coleccionado en la Fazenda Itajuru, cerca de Catas Altas $\left(20^{\circ} 5^{\prime}\right.$ S, 43 25' W), al norte de Mariana.

\section{Bibliografía}

FERNÁNDEZ, A. 1981. Recuentos cromosómicos en Malvales. Bonplandia 5(10): 63-71.

FRYXELL, P. A. 1985. Sidus sidarum. V. The North and Central American species of Sida. Sida 11(1): 62-91.

_. 1992. Malvaceae. In G. Harling \& L. Andersson, Flora of Ecuador 44: 3-142.

LAVIA, G. I., A. FERNÁNDEZ \& A. KRAPOVICKAS. 2007. Cromosomas de especies de Sida americanas (Malvaceae). Bonplandia 16(3-4): 00-00.

SCHUMANN, K. 1891. Malvaceae. In C. F. P. Martius, Flora brasiliensis 12 (3).

URBAN, I. 1893. Biographische Skizzen. 1. Friedrich Sellow (1789-1831). Bot. Jahrb. Syst. 17: 177-198.

\section{Índice de coleccionistas}

Arbo, M.M. \& al. 4012 (4)

Arbo, M.M. \& A. Schinini 2467 (7)

Baitello, J.B. 541 (1),

Barco, R. 548 (5)

Barrreto, K.D. \& al. 2185 (1),

Bartolomeu, J.G. 5198 (2)

Bastian, E. 763 (5) 
Beck, ST.G. 6208 (5)

Bernacci, L.C. \& al. 1434 (1)

Charpin, A. \& L. Ramella 21543 (5)

Ehrich, R. 394 (5)

Falkennberg, D. s.n. (7)

Ferreyra, R. 9295 (3)

Gaudichaud, Ch. 938 (2)

Gottsberger, G. 11-5268 (2), 11-20168 (2),

Hassler, E. 5121 (9)

Hatschbach, G. 15536 (9), 16151 (1), 20875 (2), 21042 (2), 31814 (1), 33530 (9), 34404 (1),

Heringer, E.P. 5599 (1), 8130/324 (1)

Hoehne, W. 3450 (1), 13449 (1), 13450 (1), 17338 (1)

Imaguire, N. 486 (1), 749 (1)

Irwin, H.S. \& al. 10710 (2)

Insp. de Plantas Texteis 11 (6)

Itaipú Internacional 811 (3)

Keller, H.A. \& al. (1)

Keller, H.A. \& F. Robledo (1)

Kirizawa, M. \& al. 3062 (1)

Klein, R.M. 955 (2)

Krapovickas, A. \& al. 23243 (2), 45470 (5), 45534 (5)

Krapovickas, A. \& C.L. Cristóbal 33449 (4), Zardini, E. M. \& al. 42391 (5)
35392 (2), 35400 (2), 35500 (1), 37221 (6), 38444 (2), 39609 (2)

Krapovickas, A., C.L. Cristóbal \& A. Schinini 45403 (5)

Leitão Filho, H.F., G. Shepherd \& W. Stubblebine \& al. 9649 (4)

Mattos, J. 31280 (7)

Monteiro, H. da C. 1200 (1)

Occhioni, P. 849 (2), 5421 (2)

Oliveira, A.M. 73 (4)

Pérez de Molas, L. \& G. Navarro 9227 (5)

Pickel, B. 5259 (1)

Rambo, P.B. 9124 (7), 9472 (7)

Rapini, A. \& al. 85 (1)

Reitz, P.R. \& R.M. Klein 6248 (2), 12263 (2), 16567 (9)

Ribas, O.S. \& al. 5126 (1)

Sampaio, A. 1965 (1)

Savassi, A.P. \& al. 268 (1)

Tressens, S.G. \& al. 3500 (8)

Tressens, S.G., M. Beccaceci \& R. Vanni 4552 (8)

Tressens, S.G., R. Vanni \& G. López 6711 (8)

Tunqui, S. 380 (3)

Original recibido el 31 de mayo de 2007; aceptado el 15 de agosto de 2007. 